\title{
Mobility analysis of a robotic cardiopulmonary resuscitation device
}

\author{
Mahdi Alinaghizadeh Ardestani ${ }^{1} *$ and Mohsen Asgari ${ }^{2}$ \\ ${ }^{\prime}$ Department of Electrical and Computer Engineering, Faculty of Shamsipour, Tehran Branch, Technical and Vocational University (TVU), \\ Tehran, Iran \\ *E-mail: ardestani@tvu.ac.ir \\ ${ }^{2}$ Robotics and Control (RC) Research Laboratory, Faculty of Mechanical Engineering, Semnan University, Semnan, Iran
}

\begin{abstract}
:
During chest compressions action, in CPR (CPR), the 2 arms of the rescuer constitute a parallel mechanism. Inspired by this performance, during this study a specific family of lower mobility parallel manipulators by employing a modified version of Delta robot is proposed for chest compressions in rescuing a patient. One of the biggest differences between this mechanism and the Delta parallel mechanism is that the position of the three active connections of the robot relative to each other has changed the geometry of the platforms. Also, it shapes the asymmetrical structure within the robot mechanism and its workspace. Another difference is due to the architectural optimization method considering the mixed performance index, which has been used during this mechanism to achieve a much better compromise between the manipulator dexterity and its workspace. Within the present paper, after introducing the architecture of the robot, a closed-form solution is developed for the kinematic problem and therefore the results are verified using MSC. Adams $\odot$. Then Jacobian matrix is generated to gauge the singularity problem of the proposed mechanism. then, the workspace of the robot is investigated and compared with the original Delta mechanism.
\end{abstract}

Keywords: Parallel Manipulators; CPR device; Singularity Analysis; Medical robots.

\section{Introduction}

Cardiopulmonary resuscitation (CPR) may be a sudden procedure that needs a quick and efficient operation. just in case of a patient is in asystole, CPR must be applied in both rescue breathing (mouthto-mouth resuscitation) and chest compressions. Generally, the chest compression frequency for an adult is at the speed of about 100 times per minute with a depth of 4 to five centimeters using two hands. Manual chest compression features a poor outcome due to some reasons like instability thanks to inertia during changes in speed during a moving environment, like an ambulance. It also consumes tons of energy and rescuer fatigue may negatively impact the standard of CPR during continuous chest compression. additionally, some patients can also be infected by other indeterminate and infelicitous diseases like Covid-19 which will cause the rescuer who had performed CPR to such patients to be infected with the infelicitous disease. Therefore, this study aimed to introduce a replacement parallel mechanism to enhance the standard of chest compression, rather than doctors, with minimal interruptions and fewer human resources. In the last three decades, parallel robots have received more attention from researchers because of their inherent benefits. A parallel manipulation is a closed-loop mechanical structure generally composed of two operating systems, which are connected in parallel by joints and legs. A parallel manipulation is a closed-loop mechanical structure consisting of two platforms, which are connected in parallel by joints and legs. One among them is attached to the fixed reference base. the opposite one can have arbitrary motion in its workspace. Several legs or moving limbs, built as serial robots, attach a moving platform to a fixed frame. Typically, the number of actuators is adequate to the number of degrees of freedom (DoFs). A previous couple of years have witnessed a crucial development within the use of those robots within the industrial $[1,2]$ and medical applications [3,4] due to their stiffness. Unlike the serial-type robot manipulators, the parallel configuration allows for a distribution of payload among their closed-loop kinematic chains. additionally, within the parallel architecture, the actuators are fixed to the bottom, so that the load of the driving force motors isn't supported by the kinematic chains. This leads to thinner and lighter kinematic chains, subsequently reducing inertia that creates a rise in both of operating speed and payload capacity of the manipulator relative to its total mass. So, these robots are expected to be costeffective from an influence requirements point of view, as compared with their serial counterparts. because the end effector is connected to more links, such a mechanism can also be very stiff and 
powerful. Accuracy and flexibility are high too, errors are averaged instead of adding up also. However, they suffer from the issues of relatively small useful workspace and these sorts of robots are usually less flexible in their use of the working volume. Another drawback (that must be taken into consideration within the design process) arises from the very fact that the natural frequencies of those systems tend to be above those of serial robots due to their smaller moving mass. The 6-DoF parallel manipulators are one among the foremost popular robots, which are studied by researchers such $[5,6]$. In these designs, the mobile platform is connected to the actuators with six links, in which case only axial loads are applied, which causes a low-motion mass and especially the robot stiffness. But they do have some tedious problems, such as design difficulties, limited workspace, and finding closed-form expressions for direct kinematics. the supply of closed-form solutions enables accurate design and efficient planning and control. Since the parallel mechanisms with but 6-DoF, such $[7,8]$, are simpler structures with fewer links and actuators, their cost is far but 6-DoF parallel manipulator with six driving limbs and are relatively easy to regulate. to avoid mentioned problems of 6-DoF parallel manipulators parallel robots with but 6-DoF, called limited-DoF manipulators which maintain the inherent advantages of parallel mechanisms and possess several other advantages like reduction of the entire cost of the device, like $[9,10]$, is attracting the attention of varied researchers. Also, among these architectures, parallel manipulators with three translational DoF, such [11], are getting increasingly popular, which are better suited for top stiffness and high-speed manipulation.There is little question that the Delta robot and its topologies are the foremost successful parallel robots for top speed applications, particularly pick-and-place of sunshine objects, e.g. food, pharmaceutical, and electronic industries. the first Delta robot is such a parallel robot that's built using parallelogram mechanisms and therefore the moving platform, which has three translational and one additional uncoupled rotational DoFs regarding the bottom invented by [12]. Then some studies have also been contributed to the linear Delta robot $[13,14]$ and reconfigurable Delta robot $[15,16]$. Various methods are applied for the kinematic model [17] and synthesis [18,19] of the Delta robot. In [20,21] Delta robot is meant for a specified workspace. Singularity analysis is an important issue for the synthesis of parallel manipulators. This property has received great attention within the literature $[22,23]$. These singular configurations are often found using analytical [24,25], numerical or geometrical methods [26,27]. A crucial limitation of the parallel manipulator is that singular configurations may exist within its workspace where the manipulator gains one or more degrees of freedom and thus loses its stiffness completely. Many researchers have categorized these singularities that support the Jacobian matrix properties of the mechanism. within the case of parallel manipulators, the Jacobian analysis may be a far more difficult problem than that of serial manipulators thanks to the existence of closed-loop kinematic chains. Generalized Jacobian analysis of lower mobility manipulators has been performed in [28]. In [29] the Jacobian matrix has been separated into two matrices: one related to the direct kinematics and therefore the other with the inverse kinematics. counting on which matrix is singular, the robot could also be at an immediate kinematics singularity, an inverse kinematics singularity, or both of them. There is much interesting research about singularity analysis of parallel manipulators and different approaches like velocity vector loop [30], screw theory [31,32], Grassmann-Cayley algebra [33,34], and Grassmann geometry method [35,36] are considered. Determination of singularities in Delta and Delta-like manipulators has been performed in [30] and [37] respectively. In [38] internal singularity of the Delta robot with articulated traveling plate has been analyzed. Concerning other related topics about Delta-type parallel robots, their modeling and control have been performed by various researchers. for instance [39] implemented modeling and control of Delta robot in ELAU GmbH hardware and software, and compared the results with the Simulink model. Index analysis of the Delta robot has been investigated in [40].

This contribution extends our previous paper [41] in which we studied and compared the feasibility of applying supervisory controller and ANFIS instead of conventional PD controller used in the C-T method without considering singularities. In that article, we assumed that the manipulator is away from singularities, but in practice, the robot is exposed to singularities. Therefore, it is necessary to find the singular configurations to avoid constituting a serious problem for tracking a planned path in the operational space.

The aforementioned architecture has utilized optimization methodology by making compromises between the performance of manipulator dexterity and workspace volume using the weighted sum of them as the mixed performance index. The architecture of the manipulator is described in detail and 
closed-form equations are developed for both the inverse and forward kinematics. Then, commercial software MSC.Adams $@$ is used to verify the closed-form results. For a given trajectory in Cartesian space, verification results are as close as possible to the closed-form results. After that, the Jacobian of the robot is derived and singularity analysis of the manipulator is performed using velocity vector-loop equations as a simple, primitive, and effective method. Finally, by considering some of the main design criteria of this robot, with the Mechanical Desktop software, the manipulator workspace is created and its asymmetrical shape is compared with the Delta original manipulator workspace.

\section{Kinematic analysis}

\subsection{Architecture description}

The schematic of the designed robotic CPR device is illustrated in Fig. 1. This mechanism has a mobile platform in the button of the robot, a fixed base at the top, and three limbs of identical RRPaR kinematic structure with the first $\mathrm{R}$ joint actuated by a rotary actuator. We use $\mathrm{Pa}$ and $\mathrm{R}$ stands for the notation of parallelogram and the revolute joint, respectively.
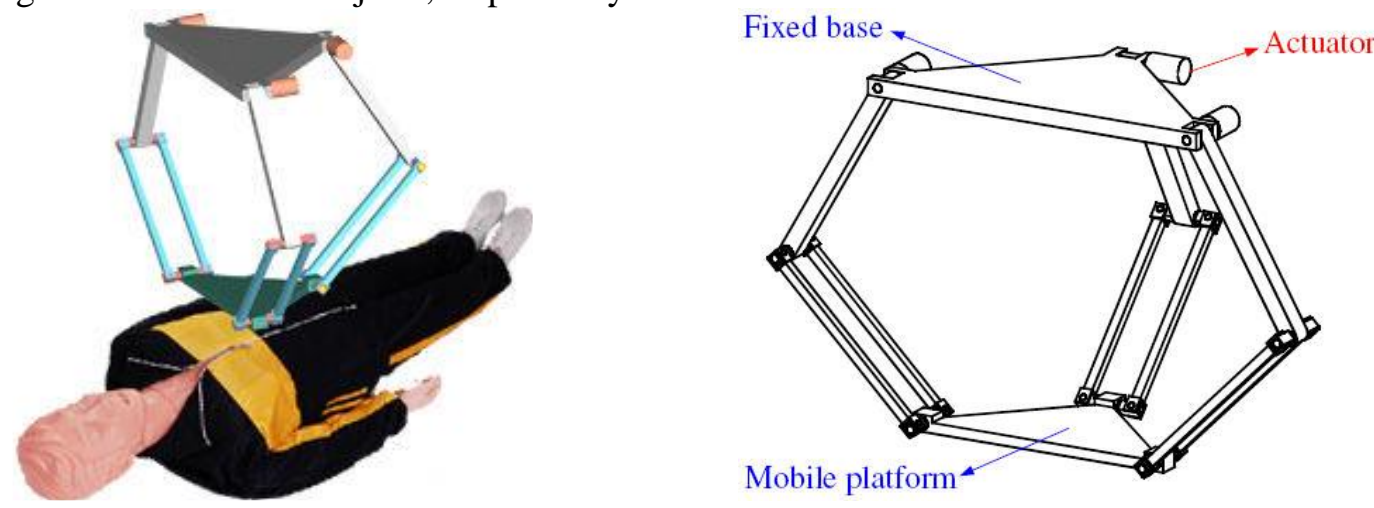

Fig. 1. The designed asymmetrical spatial parallel robot.

As shown in Fig. 2, it is assigned a fixed frame $O\{x, y, z\}$ at the point $O$ of the fixed base $\triangle A_{1} A_{2} A_{3}$. In the same way, a moving frame $P\{u, v, w\}$ at the point $P$ of the mobile platform $\Delta C_{1} C_{2} C_{3}$, with $x, y$ axis parallels to $u, v$ axis, respectively, and the $z$ and $w$ axes are perpendicular to the platform. Both the moving platform and fixed base are designed as an isosceles triangle described by the parameter of $r=$ $O A_{i}$ and $h=P C_{i}$, respectively, for $i=1,2$, and 3 . The actuated variable of the $i$ th limb is $q_{1 i}$. The connecting joints between the upper and the lower links denote by $B_{i}$. In addition, $a=A_{i} B_{i}$ and $b=B_{i} C_{i}$ are the lengths of upper and lower links of each limb respectively.

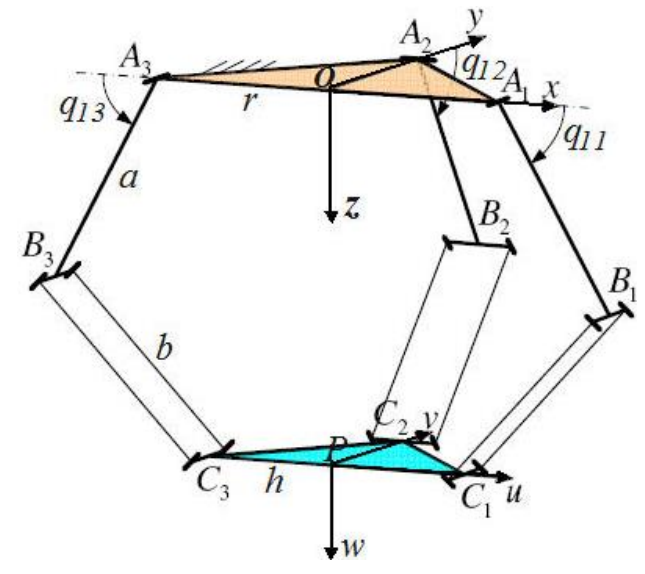

Fig. 2. Schematic representation of a 3-RRPaR parallel manipulator.

Table 1 describes the optimized architectural parameters of the mechanism.

Table 1. Architectural parameters of the robot.

\begin{tabular}{|c|c|c|}
\hline Parameter & Value & Unit \\
\hline$r$ & 160 & $\mathrm{~mm}$ \\
\hline
\end{tabular}




\begin{tabular}{|c|c|c|}
\hline$a$ & 200 & $\mathrm{~mm}$ \\
\hline$b$ & 200 & $\mathrm{~mm}$ \\
\hline$h$ & 120 & $\mathrm{~mm}$ \\
\hline
\end{tabular}

\subsection{Inverse kinematics}

In this part of the article, the inverse kinematics of the proposed parallel manipulator is considered. The joint angles related with the $i$ th limb are defined, as it is shown in Figure 3. where $p$ is the point $P$ of the mobile platform position vector. Coordinate system $\left(x_{i}, y_{i}, z_{i}\right)$ is attached to the base at point $A_{i}$ of the robot platform, such that the $x_{i}$-axis is in line with the extended line of $\overline{O A}$, the $y_{i}$-axis is directed along the revolute joint axis at $A_{i}$, and therefore the $z_{i}$-axis is parallel to the $z$-axis. The angle $\phi_{i}$, is measured from the $x$-axis to the $x_{i}$-axis and it is a constant constraint in manipulator design. Similar to the same method, $q_{1 i}$ is measured from the $x_{i}$-axis to $\overline{A B}, q_{2 i}$ is defined from the extended line of $\overline{A B}$ to the line defined by the intersection of the $x_{i}-z_{i}$ and plane of the parallelogram, and finally, $q_{3 i}$ is measured from the $y_{i}$ direction to $\overline{B_{i} C_{i}}$.

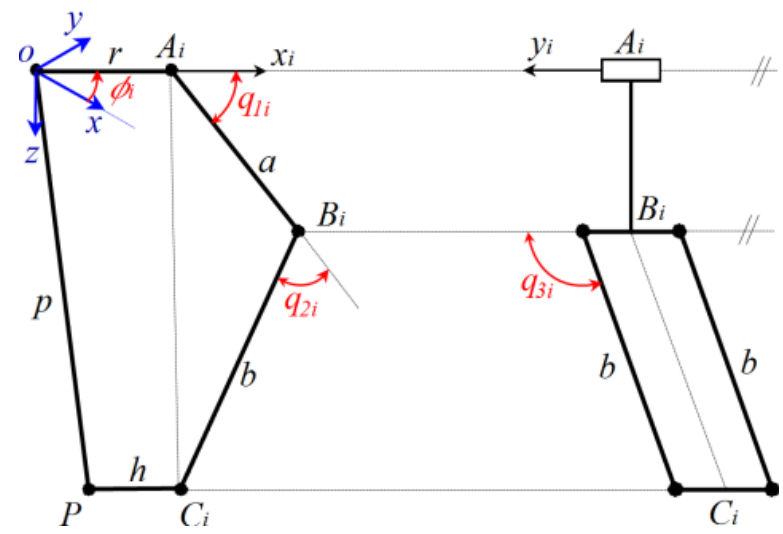

Fig. 3. Description of the joint angles.

A loop-closure equation can be written for each limb:

$\overline{A_{i} B_{i}}+\overline{B_{i} C_{i}}=\overline{O P}+\overline{P C_{i}}-\overline{O A_{i}}$

Expressing Eq. (1) in the $\left(x_{i}, y_{i}, z_{i}\right)$ coordinate frame, we obtain

$$
\left[\begin{array}{c}
a \cos q_{1 i}+b \sin q_{3 i} \cos \left(q_{1 i}+q_{2 i}\right) \\
b \cos q_{3 i} \\
a \sin q_{1 i}+b \sin q_{3 i} \sin \left(q_{1 i}+q_{2 i}\right)
\end{array}\right]=\left[\begin{array}{c}
c_{x i} \\
c_{y i} \\
c_{z i}
\end{array}\right]
$$

where

$$
\left[\begin{array}{c}
c_{x i} \\
c_{y i} \\
c_{z i}
\end{array}\right]=\left[\begin{array}{ccc}
\cos \phi_{i} & \sin \phi_{i} & 0 \\
-\sin \phi_{i} & \cos \phi_{i} & 0 \\
0 & 0 & 1
\end{array}\right]\left[\begin{array}{c}
p_{x} \\
p_{y} \\
p_{z}
\end{array}\right]+\left[\begin{array}{c}
h-r \\
0 \\
0
\end{array}\right]
$$

For inverse kinematic, the position vector $p$ of the mobile platform is given and the problem is to find the joint angles $q_{11}, q_{12}$, and $q_{13}$ required to bring the mobile platform to the desired position. By solving the second row of Eq. (2), two solutions for $q_{3 i}$ are found:

$$
q_{3 i}=\cos ^{-1} \frac{c_{y i}}{b}
$$

However, it will be shown later that both solutions for $q_{3 i}$ result similar physical position for limb $i$. After that, $q_{2 i}$ can be determined by summing the squares of Eq. (2) components.

$2 a b \sin q_{3 i} \cos q_{2 i}+a^{2}+b^{2}=c_{x i}^{2}+c_{y i}^{2}+c_{z i}^{2}$

Hence 


$$
q_{2 i}=\cos ^{-1} k
$$

where

$$
k=\left(c_{x i}^{2}+c_{y i}^{2}+c_{z i}^{2}-a^{2}-b^{2}\right) /\left(2 a b \sin q_{3 i}\right)
$$

Therefore, corresponding to each solution of $q_{3 i}$, Eq. (6) yields two solutions for $q_{2 i}$. These solutions generate four solution sets for $q_{2 i}$ and $q_{3 i}$. Additionally, according to each solution set of $q_{2 i}$ and $q_{3 i}$, Eq. (2) yields a unique solution for $q_{1 i}$. Although, all of the four solution sets contain only two distinct values of $q_{1 i}$ :

$$
q_{1 i}=2 \arctan \left(\frac{2 a c_{z i} \pm \sqrt{4 a^{2} c_{z i}^{2}-\left(c_{x i}^{2}+c_{y i}^{2}+c_{z i}^{2}+a^{2}-b^{2}\right)^{2}+\left(2 a c_{x i}\right)^{2}}}{c_{x i}^{2}+c_{y i}^{2}+c_{z i}^{2}+a^{2}-b^{2}+2 a c_{x i}}\right)
$$

According to the limb geometry and these relationships, each limb assumes the same physical position for each $q_{1 i}$. Hence, for every limb, four solution sets are realized in just two distinct positions.

\subsection{Forward kinematics}

For the forward kinematics, the input joint angles $q_{11}, q_{12}$ and $q_{13}$ are given and the problem is to find the position of the mobile platform. Substituting Eq. (3) into Eq. (2) and rearranging, we obtain

$$
\left[\begin{array}{c}
b \sin q_{3 i} \cos \left(q_{1 i}+q_{2 i}\right) \\
b \cos q_{3 i} \\
b \sin q_{3 i} \sin \left(q_{1 i}+q_{2 i}\right)
\end{array}\right]=\left[\begin{array}{ccc}
\cos \varphi_{i} & \sin \varphi_{i} & 0 \\
-\sin \varphi_{i} & \cos \varphi_{i} & 0 \\
0 & 0 & 1
\end{array}\right]\left[\begin{array}{l}
p_{x} \\
p_{y} \\
p_{z}
\end{array}\right]+\left[\begin{array}{c}
-a \cos q_{1 i}-r+h \\
0 \\
-a \sin q_{1 i}
\end{array}\right]
$$

\subsection{Verification of the obtained kinematic model}

A complex described trajectory of the mobile platform in the Cartesian space is considered for simulation studies to investigate the obtained kinematic model.

$$
\begin{aligned}
& x=-0.1 \sin (\pi t) \\
& y=0.1 \cos (\pi t) \\
& z=-0.29+0.05 \cos \left(\frac{\pi}{2} t\right)
\end{aligned}
$$

Where $t$ denotes the time variable, and $x, y$ and $z$ are in units of meters. According to the parameters of Table 1, the simulation results of the kinematic model are shown in Figs. 4, 5, and 6. These Figs are shown the joint angular displacements, velocities, and accelerations, respectively, which are derived via inverse kinematic closed-form solutions and inverse kinematics mode of MSC.Adams@ software.

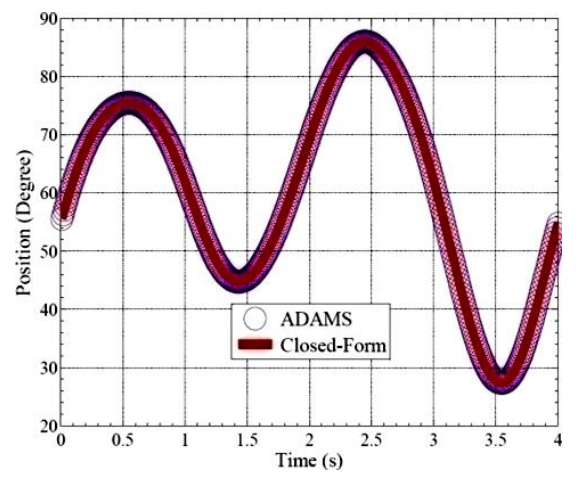

(a)

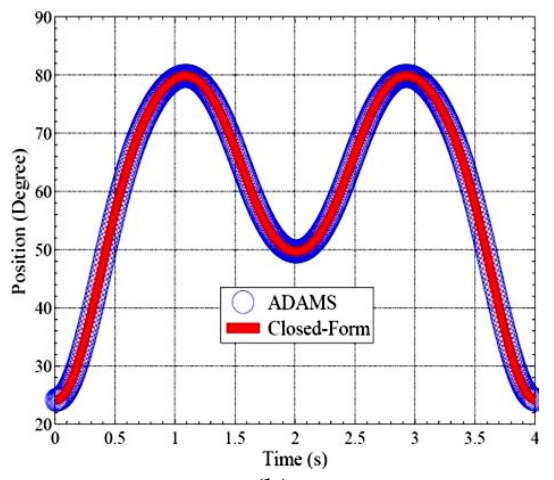

(b)

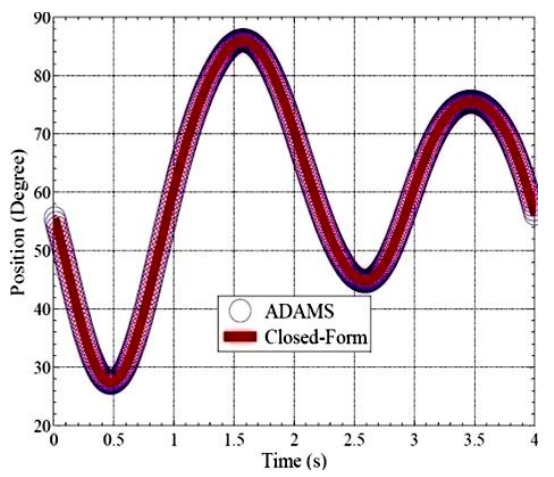

(c)

Fig. 4. Verification results for angular displacements of (a) joint 1 (b) joint 2, and (c) joint 3. 


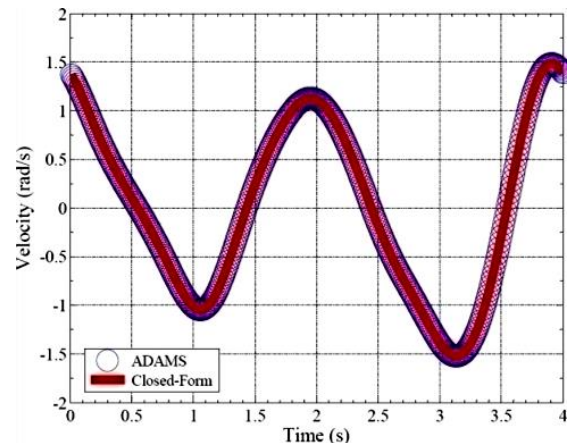

(a)

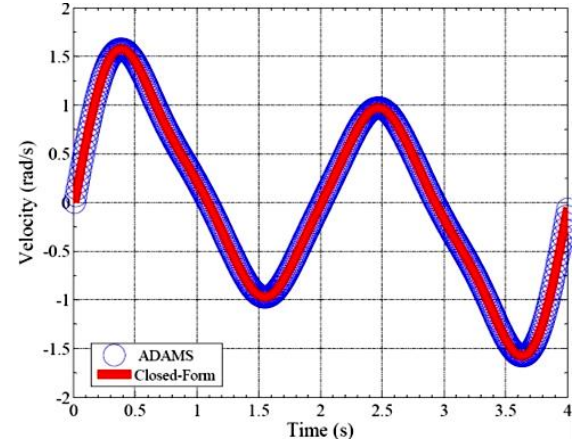

(b)

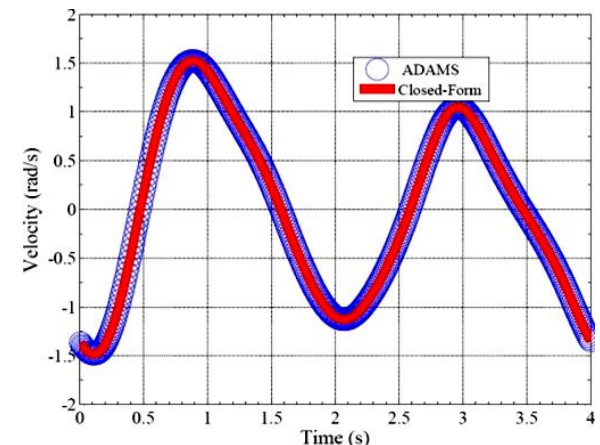

(c)

Fig. 5. Verification results for angular velocities of (a) joint 1 (b) joint 2, and (c) joint 3.

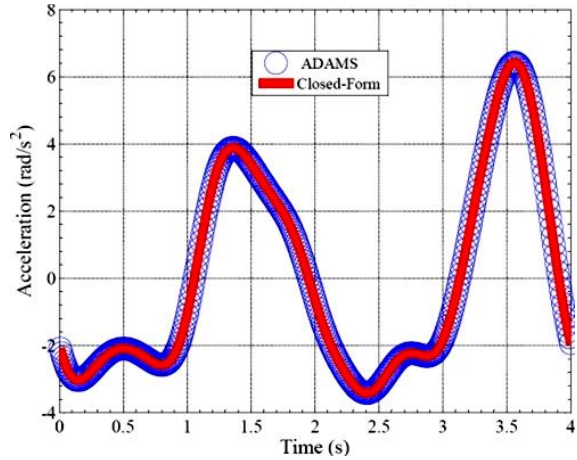

(a)

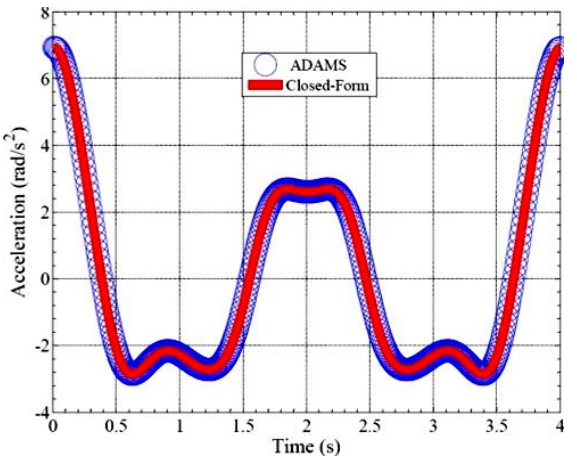

(b)

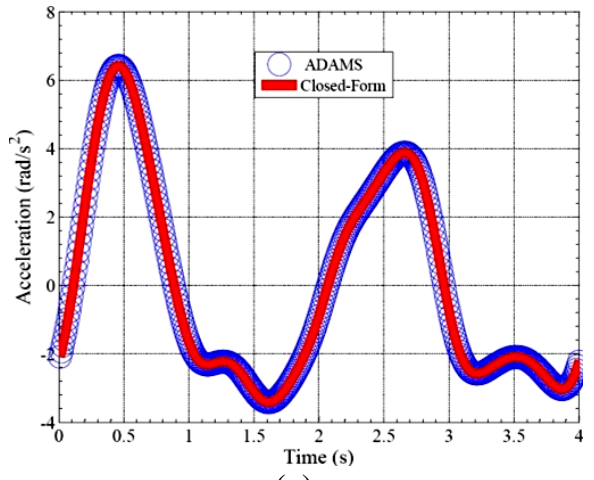

(c)

Fig. 6. Verification results for angular accelerations of (a) joint 1 (b) joint 2, and (c) joint 3.

\section{Jacobian of the manipulator}

This section is devoted to the Jacobian analysis of the robot. The kinematic constraints required by the limbs can be written in this form

$\mathbf{f}(\mathbf{p}, \mathbf{q})=0$

Differentiating (11) with respect to time leads to the relationship between the input and output speeds as follows:

$J_{p} \dot{\mathbf{p}}=J_{q} \dot{\mathbf{q}}$

where

$J_{p}=\frac{\partial \mathbf{f}}{\partial \mathbf{p}}$, and $J_{q}=\frac{\partial \mathbf{f}}{\partial \mathbf{q}}$

Let $\dot{\mathbf{p}}=[\dot{x}, \dot{y}, \dot{z}]^{\mathrm{T}}$ be the linear velocity of moving platform, and $\dot{\mathbf{q}}=\left[\dot{q}_{11}, \dot{q}_{12}, \dot{q}_{13}\right]^{\mathrm{T}}$ be the vector of the input joint velocities. A loop-closure equation (1) for $i$ th limb can be rewritten as:

$\overline{O P}+\overline{P C_{i}}=\overline{O A_{i}}+\overline{A_{i} B_{i}}+\overline{B_{i} C_{i}}$

Differentiating Eq. (13) with respect to time yields

$\dot{\mathbf{p}}=\omega_{1 i} \times \mathbf{a}_{i}+\omega_{2 i} \times \mathbf{b}_{\mathrm{i}}$,

where $\mathbf{a}_{i}=\overline{A_{i} B_{i}}, \mathbf{b}_{i}=\overline{B_{i} C_{i}}$, and $\omega_{2 i}$ is the angular velocity of the lower link of the $i$ th limb. To eliminate the passive joint rate $\omega_{2 \mathrm{i}}$, we dot-multiply both sides of Eq. (14) by $\mathbf{b}_{i}$. This gives:

$\mathbf{b}_{i} \cdot \dot{\mathbf{p}}=\omega_{1 i} \cdot\left(\mathbf{a}_{i} \times \mathbf{b}_{i}\right)$.

Expressing the vectors in Eq. (15) in the $\left(x_{i}, y_{i}, z_{i}\right)$ coordinate frame, we have 
${ }^{i} \mathbf{a}_{i}=a\left[\begin{array}{c}\cos q_{1 i} \\ 0 \\ \sin q_{1 i}\end{array}\right],{ }^{i} \mathbf{b}_{i}=b\left[\begin{array}{c}\sin q_{3 i} \cos \left(q_{1 i}+q_{2 i}\right) \\ \cos q_{3 i} \\ \sin q_{3 i} \sin \left(q_{1 i}+q_{2 i}\right)\end{array}\right],{ }^{i} \omega_{1 i}=\left[\begin{array}{c}0 \\ -\dot{q}_{1 i} \\ 0\end{array}\right],{ }^{i} \dot{\mathbf{p}}=\left[\begin{array}{c}\dot{x} \cos \varphi_{i}+\dot{y} \sin \varphi_{i} \\ -\dot{x} \sin \varphi_{i}+\dot{y} \cos \varphi_{i} \\ \dot{z}\end{array}\right]$

Substituting the expression above into Eq. (15), after simplification we obtain

$j_{i x} \dot{x}+j_{i y} \dot{y}+j_{i z} \dot{z}=a \sin q_{2 i} \sin q_{3 i} \dot{q}_{1 i}$.

Where

$j_{i x}=\cos \left(q_{1 i}+q_{2 i}\right) \sin q_{3 i} \cos \varphi_{i}-\cos q_{3 i} \sin \varphi_{i}$

$j_{i y}=\cos \left(q_{1 i}+q_{2 i}\right) \sin q_{3 i} \sin \varphi_{i}-\cos q_{3 i} \cos \varphi_{i}$

$j_{i z}=\sin \left(q_{1 i}+q_{2 i}\right) \sin q_{3 i}$.

Writing Eq. (16) for $i=1,2$, and 3, results in three scalar equations, which can be assembled in matrix form as

$J_{p} \dot{\mathbf{p}}=J_{q} \dot{\mathbf{q}}$

Where

$J_{p}=\left[\begin{array}{lll}J_{1 x} & J_{1 y} & J_{1 z} \\ J_{2 x} & J_{2 y} & J_{2 z} \\ J_{3 x} & J_{3 y} & J_{3 z}\end{array}\right], J_{q}=a\left[\begin{array}{ccc}\sin q_{21} \sin q_{31} & 0 & 0 \\ 0 & \sin q_{22} \sin q_{32} & 0 \\ 0 & 0 & \sin q_{23} \sin q_{33}\end{array}\right]$

\section{Singularity analysis}

Due to the existence of two Jacobian matrices, which are derived in the previous section, the robot imposes a singular condition when either $J_{p}$ or $J_{q}$ or both of them are singular. Additionally, there also exists another kind of singularity. This type of singularity arises from reducing a certain number of legs from the full kinematic chain and carrying out a Jacobian analysis for the reduced loop. The corresponding Jacobians are termed as the intermediate Jacobians (Lopez et al., 2006).

\subsection{Inverse kinematic singularities}

These kinds of singularities are related to the inverse Jacobian, $J_{q}$, from Eq. (17), and occur when the following condition is verified:

$\operatorname{det}\left(J_{q}\right)=0$.

So Eq. (18) implies

$q_{2 i}=0$ or $\pi$,

or

$q_{3 i}=0$ or $\pi$,

The condition (19) occurs when the upper and lower link are in the same plane. The condition (20) occurs when all the links of the parallelogram of a limb are parallel or anti-parallel to $y$-axis. It means that they are collinear. In other words, in this posture $a_{i} \perp b_{i}$. These configurations occur at the workspace upper and lower boundaries. Fig. 7 shows these positions. 


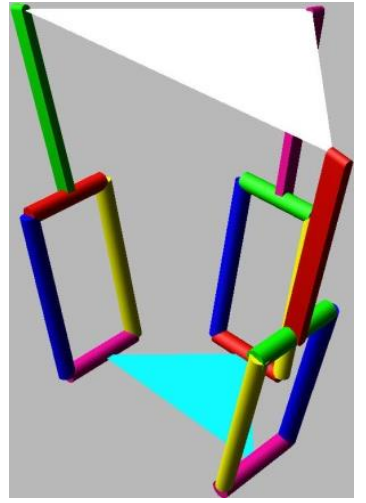

(a)

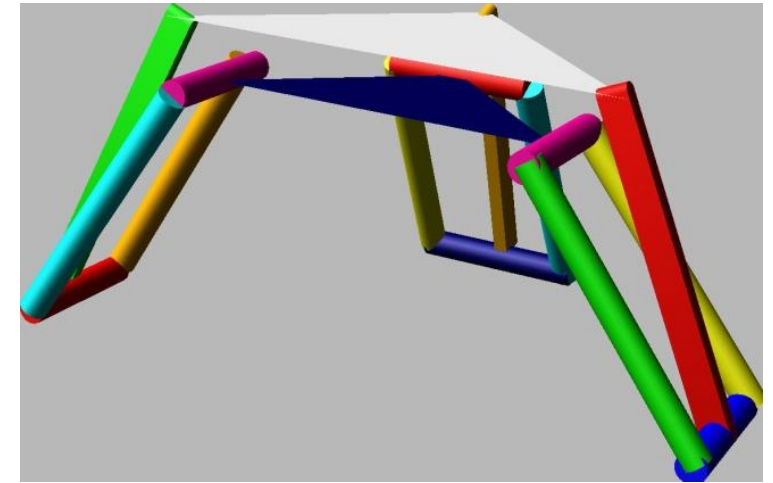

(b)

Fig. 7. Inverse kinematic singularities (a) Completely stretched, and (b) Completely contracted configuration.

\subsection{Forward kinematic singularities}

Forward kinematic singularities are associated with the forward Jacobian, $J_{p}$, from Eq. (17), and arises corresponds to the following condition:

$\operatorname{det}\left(J_{p}\right)=0$.

These types of singularities are much more complicated than the inverse kinematic singularities due to their complicated Jacobian. Although it is difficult to solve for all possible singularities, several postures satisfy this condition. $J_{p}$ becomes singular for some real value of $\mu_{1}, \mu_{2}$, and $\mu_{3}$ (where not all $\mu$ 's are zero) when:

$\mu_{1} \mathbf{j}_{1}+\mu_{2} \mathbf{j}_{2}+\mu_{3} \mathbf{j}_{3}=0$

where each row of $J_{p}$ represents a unit vector, $\mathbf{j}_{i}=\left[j_{i x}, j_{i y}, j_{i z}\right]^{\mathrm{T}}$, which points along the direction of a lower link. One condition that satisfies Eq. (22) is found when all three unit vectors lie on a plane. For some nonzero value of $\mu_{1}, \mu_{2}$, and $\mu_{3}$ from the first two equations in terms of manipulator variables, this condition can be written as

$\sin \left(q_{11}+q_{21}\right) \sin q_{31}=\sin \left(q_{12}+q_{22}\right) \sin q_{32}=\sin \left(q_{13}+q_{23}\right) \sin q_{33}=0$.

Eq. (23) indicates singular configurations when

$q_{1 i}+q_{2 i}=0$ or $\pi$,

or

$q_{3 i}=0$ or $\pi$,

Condition (24) implies that the third column of $J_{p}$ is zero. Physically, the geometrical interpretation of this condition is that all the lower links are in the plane of the moving platform and they can have both $x_{i}$ and $y_{i}$ components non-zero.

The condition (25) is a combined singularity and it also implies that the third column of $J_{p}$ is zero. Physically, the posture of the manipulator again corresponds to when all the lower links are in the same plane as the moving platform and lie entirely along $y_{i}$-axes. Fig. 8 shows this type of singularity. 


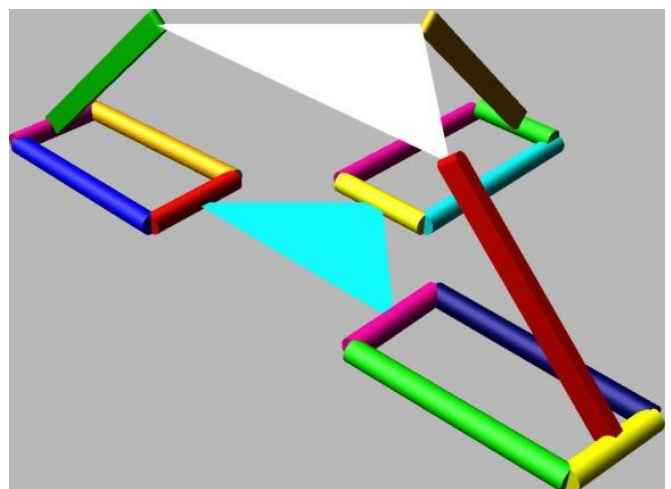

Fig. 8. A singular configuration in which all the lower links are in the plane of the moving plate.

The second set of postures that satisfies Eq. (22) is found by the following condition

$\mathbf{j}_{i}= \pm \mathbf{j}_{k} \quad$ for $\quad i \neq k$

In this singular configuration, any two of the lower links are parallel to each other. Fig. 9 shows such a singular configuration.

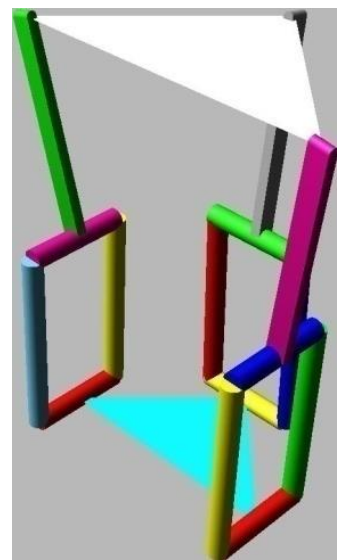

Fig. 9. Singular configuration in which two or three lower links are parallel to each other.

\subsection{Intermediate Jacobians and singularities}

In this section, intermediate Jacobians find the relation between the velocity of the end-effector and the time rate of change of the length of a kinematic chain. This idea was introduced in (Lopez et al., 2006). To apply this idea for the modified Delta mechanism, the closed-loop $O A_{i} C_{i} P \subseteq O A_{i} B_{i} C_{i} P$, which is obtained by ignoring the limbs $a$ and $b$ in the full kinematic chain $O A_{i} B_{i} C_{i} P$, is reviewed. A loop-closure equation (1) for $i$ th limb again can be rewritten as:

$\overline{A_{i} C_{i}}=\overline{O P}+\overline{P C_{i}}-\overline{O A_{i}}$

Writing the $\overline{A_{i} C_{i}}$ components in the reference frame $x_{i} y_{i} z_{i}$, then by simplifying the resulting equation leads to

$\left(A_{i} C_{i}\right)^{2}=\left(A_{i} C_{i}\right)_{x}^{2}+\left(A_{i} C_{i}\right)_{y}^{2}+\left(A_{i} C_{i}\right)_{z}^{2}=\left(p_{x}+(h-r) \cos \varphi_{i}\right)^{2}+\left(p_{y}-(h-r) \sin \varphi_{i}\right)^{2}+p_{z}^{2}$

Let $f\left(p, A_{i} C_{i}\right)$ be defined as follows

$f\left(p, A_{i} C_{i}\right)=\left(p_{x}+(h-r) \cos \varphi_{i}\right)^{2}+\left(p_{y}-(h-r) \sin \varphi_{i}\right)^{2}+p_{z}^{2}-\left(A_{i} C_{i}\right)^{2}$

Let $n_{i}=A_{i} C_{i}$ and $p_{x}=p_{1}, p_{y}=p_{2}, p_{z}=p_{3}$. Differentiating Eq. (28) with respect to time yields 
$\frac{\partial f_{i}}{\partial n_{j}} \frac{d n_{j}}{d t}+\frac{\partial f_{i}}{\partial p_{j}} \frac{d p_{j}}{d t}=0$

Eq. (29) can be written in the following form

$J_{i j}^{n} \dot{n}_{j}=J_{i j}^{p} \dot{p}_{j}$

Where the intermediate Jacobian for this modified mechanism can be written into a matrix form as follows

$J_{i j}^{n}=-\left[\begin{array}{lll}\frac{\partial f_{1}}{\partial n_{1}} & \frac{\partial f_{1}}{\partial n_{2}} & \frac{\partial f_{1}}{\partial n_{3}} \\ \frac{\partial f_{2}}{\partial n_{1}} & \frac{\partial f_{2}}{\partial n_{2}} & \frac{\partial f_{2}}{\partial n_{3}} \\ \frac{\partial f_{3}}{\partial n_{1}} & \frac{\partial f_{3}}{\partial n_{2}} & \frac{\partial f_{3}}{\partial n_{3}}\end{array}\right], \quad J_{i j}^{p}=\left[\begin{array}{lll}\frac{\partial f_{1}}{\partial p_{1}} & \frac{\partial f_{1}}{\partial p_{2}} & \frac{\partial f_{1}}{\partial p_{3}} \\ \frac{\partial f_{2}}{\partial p_{1}} & \frac{\partial f_{2}}{\partial p_{2}} & \frac{\partial f_{2}}{\partial p_{3}} \\ \frac{\partial f_{3}}{\partial p_{1}} & \frac{\partial f_{3}}{\partial p_{2}} & \frac{\partial f_{3}}{\partial p_{3}}\end{array}\right]$

Where evaluating the partial derivatives in Eq. (31) lead to

$J_{i j}^{p}=\left[\begin{array}{ccc}n_{1} & 0 & 0 \\ 0 & n_{2} & 0 \\ 0 & 0 & n_{3}\end{array}\right], \quad J_{i j}^{n}=\left[\begin{array}{lll}p_{1}+(h-r) \cos \varphi_{1} & p_{2}-(h-r) \sin \varphi_{1} & p_{3} \\ p_{1}+(h-r) \cos \varphi_{2} & p_{2}-(h-r) \sin \varphi_{2} & p_{3} \\ p_{1}+(h-r) \cos \varphi_{3} & p_{2}-(h-r) \sin \varphi_{3} & p_{3}\end{array}\right]$

$J_{i j}^{n}$ and $J_{i j}^{p}$ are inverse and forward intermediate Jacobian which arises by considering sub-loop $O A_{i} C_{i} P$ of the full kinematic chain $O A_{i} B_{i} C_{i} P$. The intermediate inverse kinematic singularities imply that

$n_{i}=0$ for any of the $i$

This posture corresponds to condition (19), in which the upper links are in the plane of the lower links. The intermediate forward kinematic singularities occur when

$p_{z}=0$

or

$r=h$

In the case of the modified Delta mechanism, according to Table 1, since the lengths of upper and lower links are the same, it can be seen that condition (34) includes both the conditions (24) and (25). Also, it is observed that the condition (35) is unrelated to the configuration of the manipulator and it is associated with the structure of the mechanism corresponds to identical dimensions of the fixed base and the moving platform. Therefore, this type of singularity is not only architecture-dependent but also configuration-dependent. Note that for this modified version of the Delta robot with special kinematic architecture and parameters, both the intermediate and conventional Jacobian gave the same singular conditions. However, due to the reducing a certain number of legs from the full kinematic chain and consequently the existence of a reduced loop, the analysis of the intermediate Jacobians is easier than conventional Jacobian to arrive at these singular situations.

\subsection{Combined singularities}

This type of singularity occurs when the determinant of $J_{p}$ and $J_{q}$ are both zero. For this improved mechanism, a combined singularity occurs when the next two conditions are satisfied simultaneously: (1) the geometry of the moving platform is identical to that of the fixed base and (2) all three upper links are perpendicular to the fixed base, i.e., $\overline{A_{i} B_{i}} \perp \overline{O A_{i}}$ for $i=1$, 2, and 3. The moving platform gains 2 degrees of freedom in this configuration. Point $P$ can be positioned anywhere on a spherical surface centered at a distance $a$ above the centroid of the fixed base, when the upper links are completely locked. 
On the other hand, by keeping the moving platform steady, high links can create some extremely small rotations. Similar to condition (35) in intermediate forward kinematic singularities, the combined singularity is not only configuration dependent but also architecture-dependent. Since for this modified mechanism, the geometry of the moving platform is not the same as the fixed base, so this kind of singularity does not occur in this mechanism.

\section{Workspace Determination of Mechanism}

As mentioned earlier, the main problem of parallel manipulators is their small useful workspace. Parallel manipulator design for the desired workspace has more challenging problems. Therefore, it is expected that the architectural parameters of a parallel manipulation will be optimized to achieve the maximum volume of workspace. Many performance criteria have been proposed for the problem of the optimal design of parallel manipulators. Most of them focus only on optimizing one of the main features of the manipulator, such as [42,43]. However, a manipulator designed to maximize workspace may not be the optimal design for practical applications, and a parallel manipulation optimized for the workspace may lead to a manipulator with undesirable kinematic characteristics such as poor dexterity. Therefore, a single objective function consideration may not yield an acceptable design. To overcome this challenge, the objective function is defined as a mixed performance index, which is a weighted sum of the workspace volume index $\left(\eta_{s}\right)$, and the global dexterity index (GDI), $\left(\eta_{d}\right)$ which is given by [44] as $G D I=\frac{\int_{V}\left(\frac{1}{k}\right) d V}{V}$

where $k$ is the condition number of the Jacobian and $V$ denotes the total workspace volume.

$\eta=\left(1-w_{d}\right) \eta_{s}+w_{d} \eta_{d}=\left(1-w_{d}\right) \frac{V}{V^{*}}+w_{d}\left(\frac{1}{V} \int_{V} \frac{1}{k} d V\right)$

where the weighted parameter $w_{d}\left(0 \leq w_{d} \leq 1\right)$ describes the proportion of global dexterity index in the mixed index, and $V^{*}$ represents the robot size, which is evaluated by the product of the area of the fixed platform and the maximum reach of the moving platform in the $z$ direction.

Condition number of the Jacobian matrix $J$ is defined as

$$
k=\|J\|\left\|J^{-1}\right\|
$$

where $\|\bullet\|$ denotes the 2-norm of the matrix.

we first consider the surface composed of all possible positions of $P$ for limb $i$ with a given $q_{1 i}$, To determine the workspace of our parallel mechanism. The surface is a sphere centered at $B_{i}$. Next, we consider all three limbs. Point $P$ must fall simultaneously on the three spheres created by any sweep of $P$ for each limb. The robot's workspace is represented by the intersections of these three spheres. The generated workspace, which is specified using Mechanical Desktop software, is shown in Figure 10. 


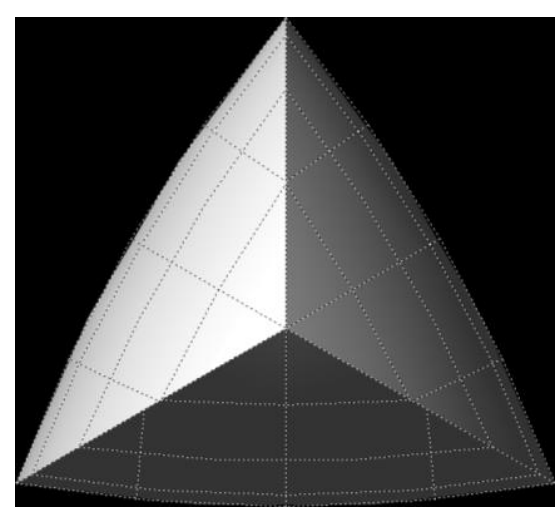

(a)

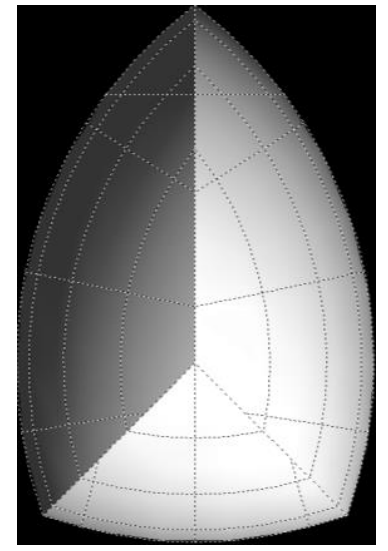

(b)

Fig. 10. Top view of the workspace of (a) original Delta, and (b) modified Delta robot.

Fig. 10(a) and 10(b) show the top view of the workspace of the original Delta, and modified Delta mechanism respectively with architectural parameters which have been mentioned in Table 1 . As it is seen in Fig. 10, compared with the original Delta mechanism, the obtained workspace has an asymmetrical character and its longitudinal direction is more extended than its lateral direction. This asymmetrical characteristic is affected by changing the position of three active joints relative to each other and it can be utilized in many applications with the special maneuver.

\section{Conclusions}

In this paper, we aimed to design a reliable device alternative to manual CPR because of the requirements from medical aspects which can reduce the risk in rescuing patients significantly. The proposed device is a novel 3DoF translational parallel manipulator by employing the architecture of a modified version of the Delta robot family. Based on the geometrical method, the closed-form solutions for both the inverse and direct kinematics problems have been developed and the results were verified using MSC.Adams $\odot$. Then singularity analysis was performed. In addition, the concept of the intermediate Jacobian developed by [30] was employed, which is much less intricate to evaluate and contains not only the information found in traditional Jacobian matrices but also describes structural singularities. Consequently, as a confirmation, it was shown that for this modified mechanism, part of the intermediate singularity includes inverse and direct singularity conditions. It should be underlined that practical purposes are needed to take into consideration these kinds of singularities. Finally, the achievable workspace was obtained using the mechanical desktop software and it was compared to the working space of the original Delta robot.

\section{References}

1. LU, YI, et al., 2010, Simulation of normal machining of 3D free-form surface by an orthogonal 3-leg parallel machine tool with 5-DOF, Computers \& Industrial Engineering, 59, 4, 764-769

2. WUJ, WANG J, LI. T, et al., 2007, Performance analysis and application of a redundantly actuated parallel manipulator for milling, Journal of Intelligent and Robotic Systems, 50, 2, 163-180

3. JAmwal PK, SQ XIE, YH TsOI, KC Aw, 2010, Forward kinematics modeling of a parallel ankle rehabilitation robot using modified fuzzy inference, Mechanism and Machine Theory, 45, 1537-1554

4. YoON, JUNGWON, et al., 2010, A 6-DOF gait rehabilitation robot with upper and lower limb connections that allows walking velocity updates on various terrains, IEEE/ASME Transactions on Mechatronics, 15, 2, 201215

5. Khalil W., GuEgan S., 2004, Inverse and direct dynamic modeling of Gough-Stewart robots, IEEE Transactions on Robotics and Automation, 20,754-762

6. Li, H., Gosselin, C. \& RichaRD, M., 2007, Determination of the maximal singularity-free zones in the sixdimensional workspace of the general gough-stewart platform, Mechanism and Machine Theory, 42, 4, 497511

7. Meng, Jian, GuAnfeng LiU, AND ZeXiang Li., 2007, A geometric theory for analysis and synthesis of sub-6 DOF parallel manipulators, IEEE Transactions on Robotics, 23, 4, 625-649 
8. ZhaO, YongJie, 2012, Dynamic optimum design of a three translational degrees of freedom parallel robot while considering anisotropic property, Robotics and Computer-Integrated Manufacturing,100-112

9. Wang, XiAoyu, LuC Baron, And Guy Cloutier, 2008, Topological and geometrical synthesis of threedegree-of-freedom fully parallel manipulators by instantaneous kinematics, Journal of Mechanical Design, 130

10. Hernández, Alfonso, et al., 2008, Transitions in the velocity pattern of lower mobility parallel manipulators, Mechanism and Machine Theory, 43, 6, 738-753

11. ZhaO, YONGJIE., 2012, Dimensional synthesis of a three translational degrees of freedom parallel robot while considering kinematic anisotropic property, Robotics and Computer-Integrated Manufacturing,169-179

12. Clavel R, 1988, DELTA, A fast robot with parallel geometry, 18th Int. Symposium on Industrial Robot, Lausanne.

13. ZENEBE, KASSA ZELALEM, 2013, Formulation of a Generalized Jacobian and Inverse Acceleration Analysis of a Linear Delta Manipulator Based on Reciprocal Screws Method, International Journal of Engineering, 2 , 2

14. M. Stock And K. Miller, 2003, Optimal Design of Spatial Parallel Manipulators: Application to linear Delta Robot, ASME Journal of Mechanical Design, 125, 292-301

15. BRISAN C. AND CSISZAR A., 2011, Computation and analysis of the workspace of a reconfigurable parallel robotic system, Mechanism and Machine Theory, 46, 1647-1668

16. Maya, Mauro, et al., 2013, Workspace and Payload-Capacity of a New Reconfigurable Delta Parallel Robot, Int. J Adv Robotic Sys., 56,10, 1-11

17. OpL, M., et al., 2012, DELTA-Robot with Parallel Kinematics, Mechatronics, Springer Berlin Heidelberg, $445-452$

18. ZhANG, Limin, et al., 2012, Dimensional synthesis of the delta robot using transmission angle constraints, Robotica, 30, 3, 343-349

19. M.A. Laribi, L. Romdhane And S. Zeghloul, 2008, Advanced Synthesis of the DELTA Parallel Robot for a Specified Workspace, Parallel Manipulators, towards New Applications, InTech, 207-225

20. X. LiU And J. WANG, 2004, A New Approach to the Design of a DELTA Robot with a Desired Workspace, Journal of Intelligent and Robotic Systems, 39, 209-225

21. Laribi, M., Romdhane, L. \& Zeghloul, S., 2007, Analysis and dimensional synthesis of the delta robot for a prescribed workspace, Mechanism and Machine Theory, 42, 859-870

22. Conconi, Michele, And Marco CARricato, 2009, A new assessment of singularities of parallel kinematic chains, IEEE Transactions on Robotics, 25, 4, 757-770

23. Macho, E., Altuzarra, O., Amezua, E. \& Hernandez, A., 2009, Obtaining configuration space and singularity maps for parallel manipulators, Mechanism and Machine Theory, 44, 11, 2110-2125

24. ZHAO, Y. J., 2013, Singularity, isotropy and velocity transmission evaluation of a three translational degrees of freedom parallel robot, Robotica, 31, 2, 193 - 202

25. Hubert J, Merlet J P, 2009, Static of parallel manipulators and closeness to singularity, ASME Journal of Mechanisms and Robotics, 1, 1

26. ZHAO J, LI B, YANG X, YU H, 2009, Geometrical method to determine the reciprocal screws and applications to parallel manipulators, Robotica, 27, 929-940

27. G. LIU, Y. LOU AND Z. LI, 2003, Singularities of parallel manipulators: A geometric treatment, IEEE Transactions on Robotics and Automation, 19, 4, 579-594

28. Huang T, Liu H T, Chetwynd, D. G., 2011, Generalized Jacobian analysis of lower mobility manipulators, Mechanism and Machine Theory, 46, 831-844

29. Gosselin, Clement, And Jorge Angeles, 1990, Singularity analysis of closed-loop kinematic chains, IEEE Transactions on Robotics and Automation, 6, 3, 281-290

30. López, M., et al., 2006, Delta robot: inverse, direct, and intermediate Jacobians, Proceedings of the Institution of Mechanical Engineers, Part C: Journal of Mechanical Engineering Science, 220, 1, 103-109

31. YU J J, DAI J S, ZHAO T S, et al., 2009, Mobility analysis of complex joints by means of screw theory, Robotica, 27, 6, 916-927

32. K. Xianwen And C. M. Gosselin, 2004, Type synthesis of 3T1R 4-dof parallel manipulators based on screw theory, IEEE Transactions on Robotics and Automation, 20, 2, 181-190 
33. Wenger, P., Caro, S., Chablat, D., 2009, Singularity Analysis of Lower Mobility Parallel Manipulators Using Grassmann-Cayley Algebra, IEEE Transactions on Robotics, 25 , 5, 995 - 1004

34. Amine, SemaAn, et al., 2012, Singularity analysis of the H4 robot using Grassmann-Cayley Algebra, Robotica, 30, 7, 1109-1118

35. P. Ben-Horin AND P. ShOHAM, 2009, Application of Grassmann-Cayley Algebra to Geometrical Interpretation of Parallel Robot Singularities, International Journal of Robotics Research, 28, 1, 127-141

36. AmINe, SEMAAN, et al., 2012, Singularity analysis of 3T2R parallel mechanisms using Grassmann-Cayley algebra and Grassmann geometry, Mechanism and Machine Theory, 52, 326-340

37. R. Di Gregorio, 2004, Determination of Singularities in Delta-Like Manipulators, The International Journal of Robotics Research, 23, 1, 89-96

38. KRUT, SÉBASTIEN, AND FrANÇOIS PIERRot., 2006, Internal singularity analysis of a class of lower mobility parallel manipulators with articulated traveling plate, IEEE Transactions on Robotics, 22, 1, 1-11

39. Olsson A., 2009, Modeling and control of a Delta -3 robot, Master's Thesis, Lund University, Lund Sweden

40. Hsu, K. S., et al., 2004, Modelling and index analysis of a Delta-type mechanism, Proceedings of the Institution of Mechanical Engineers, Part K: Journal of Multi-body Dynamics, 218, 3, 121-132

41. Asgari Mohsen, and Mahdi A. Ardestani, Dynamics and improved computed torque control of a novel medical parallel manipulator: applied to chest compressions to assist in cardiopulmonary resuscitation, $J$ Mech Med Biol 15: 1550051, 2014.

42. Silva V. G., et al., 2014, Dexterity Optimization of a Three Degrees of Freedom DELTA Parallel Manipulator, InROBOT2013: First Iberian Robotics Conference, 719-726

43. Sergiu-Dan Stan, et al, 2011, Performance analysis of 3 DOF Delta parallel robot, Proc. 4th Int. HIS Conference, Yokohama

44. Gosselin C., Angeles J., 1991, A global performance index for the kinematic optimization of robotic manipulators, ASME J. Mech. Des., 113, 3, 220-226 